\title{
EDITORIAL
}

\section{Dietary assessment at the end of life's spectrum}

European Journal of Clinical Nutrition (2009) 63, S1-S4; doi:10.1038/ejcn.2008.58

\section{Introduction}

Dietary assessment is a formidable challenge for any researcher and this is particularly the case for assessment in the young and in older people. The advantages and disadvantages of the various methods of dietary assessment are well known. The inherent challenges of dietary assessment, which may include reliance on memory, a developed sense of time, literacy, mathematical ability, knowledge of food groups, food identification and portion estimation are even more of an issue in the extremes of age of the population. This is compounded by the probability of others being responsible for food purchase and preparation.

The assessment of diet in children and older people is a key aspect of life-course determinants of health. In addition, the rise in childhood obesity and associated morbidities has made the investigation of potential dietary causes a priority. Older people are an increasingly heterogeneous population with respect to lifestyle, health and well-being; the study of these variations with respect to diet is important. Although the core methods for the measurement of diet have remained unchanged for over 60 years, advances in technology have led to substantial changes in the media available to deliver, collect and analyse dietary assessments. This is a field that is certain to continue to move apace.

The PHSRN funded workshop entitled 'Dietary assessment at the end of life's spectrum' held at Sidney Sussex College, Cambridge on 13 December 2007, sought to bring together researchers who are familiar with dietary assessment, and a line up of speakers who are experts in the field and had particular experience in the assessment of diet in young and older people. The workshop had the following aim and objectives:

\section{Aim}

To share expertise in dietary assessment methodology in young children and older people.

\section{Objectives}

- Elucidate good practices in dietary assessment in children and older people;
- Inform researchers on recent developments and advances in the field;

- Provide a forum to discuss topical issues and develop an action list of research needs.

This supplement of the European Journal of Clinical Nutrition comprises papers from the presentations at the workshop and the write-ups from the discussion sessions. An overview of the workshop programme is provided below.

\section{Main presentations}

- Overview of dietary assessment in children and the elderly Dr Ashley Adamson, Newcastle University, UK

- Cognitive processes in children's recall Dr Suzanne Domel Baxter, University of South Carolina, US

- Cognitive processes in elderly recall; FFQ use in the Scottish Mental Health Survey

Dr Geraldine McNeill, University of Aberdeen, UK

\section{Short presentations}

- Dietary assessment in the ALSPAC Study Dr Pauline Emmett, University of Bristol, UK

- Estimation of portion sizes in children's dietary assessment Dr Emma Foster, Newcastle University, UK

- Use of technology in children's dietary assessment Dr Carol Boushey, Purdue University, US

- Dietary assessment in the elderly; experience from the Boyd Orr Cohort Dr Maria Maynard, MRC Social and Public Health Sciences Unit, Glasgow, UK

- Dietary assessment in the elderly; a Swedish perspective Dr Elisabet Rothenberg, Gothenberg University

- Dietary assessment in the elderly; experience from the HALE and SENECA Studies Dr Jeanne de Vries, Wageningen University, the Netherlands

\section{Discussion topics}

- Use of technology in dietary assessment Dr Carol Boushey, Purdue University, US 
- Novel approaches to portion size estimation Dr Wendy Wrieden, University of Dundee, UK

- Working with surrogate reporters Dr Pauline Emmett, University of Bristol, UK

In the opening presentation Dr Ashley Adamson (Adamson et al., 2009) highlighted the need for researchers to be clear at the outset about their research questions and what exactly they want to glean from the data resulting from dietary assessment. The budget will ultimately determine the method used; a low-budget method will generally produce low-quality dietary data. In her research, Dr Adamson has tackled dietary assessment in young and older people and has developed new tools for this purpose. The Food Assessment in School Tool (FAST) is a simple and concise tool for assessing diet in 3- to 7-year olds and is designed for use by non-specialists. Its aim is to assess fruit and vegetable intake, general eating patterns and diet as a whole. It is a prospective method in which observers mark foods eaten from a pre-determined food list; the food list is based on the most recent National Diet and Nutrition Survey in this age group. Early results suggest that FAST is an accurate measure of fruit and vegetable intake but requires further development to measure total energy intake when compared with weighed intakes and the ratio of reported energy intake to estimated resting metabolic rate. Multiple-pass 24-h recalls repeated four times were used for the first time in a UK national survey recently, the Low Income National Diet and Nutrition Survey. Dr Adamson presented some preliminary data using a single multiple-pass recall in a cohort study in the elderly, 'The Newcastle 85 cohort'. Although more costly than a food frequency questionnaire (FFQ), the method seems to produce more accurate data when compared with a well known FFQ - the EPIC FFQ, and is feasible in this population group.

Dr Suzanne Domel Baxter presented a summary of her research on the cognitive aspects related to the recall of dietary intake in children (Baxter, 2009). Her studies have focused on detailed aspects of the recall process using a validated (observed intake) approach. Dr Baxter noted the high intra- and inter-individual variability in children's recall ability, which makes the assessment particularly challenging. A sex differential is apparent in the use of prompts, with boys responding better to reverse order (evening to morning) than girls who appeared to do better in forward order (morning to evening) prompts. One study suggested that accuracy was not reduced by telephone rather than in person interviews. Another study suggested that changing the target period to the previous $24 \mathrm{~h}$ rather than the previous day's recall significantly increased accuracy of recall. These results suggest that researchers need to give thought to the interview time and target period when planning their research to maximize accuracy. Dr Baxter's work has found similar patterns of underreporting in overweight children to that found in the adult literature. Dr Baxter outlined potential correlates of children's recall accuracy, which included social desirability, self-esteem/ body image, BMI, sex, race/ethnicity and memory/cognitive ability.

Dr Geraldine McNeill outlined the challenges of dietary assessment in the elderly based on her experience in a number of cohort studies (McNeill et al., 2009). Age-related cognitive decline can affect short-term memory and therefore may impact on the ability to undertake dietary assessment, which is a relatively complex task regardless of the method. However, there are certain advantages of undertaking dietary assessment in this population, many of whom are not affected by cognitive decline; advantages include relatively stable food habits, often less demands on time and lower dependency on food prepared outside the home. The Scottish Collaborative Group FFQ has been modified and simplified for use in older people. Results of the testing phase showed acceptable reproducibility with a lower degree of accuracy than in the younger populations, but many nutrients showed acceptable validity when compared with weighed intakes. The deleterious effect of impaired cognition was apparent. A point that is particularly relevant to the attempts to study the relationship between diet and cognitive decline. Some studies in this population group have included a measure of cognitive function and the inclusion of such an assessment was investigated as a potential confounder at the analysis stage. Cognitive function tests can present problems when respondents cheat or practise to increase their score.

In a series of short presentations, experiences of undertaking dietary assessment in the young and elderly were presented and some novel approaches to the collection of dietary intake data were cited. Dr Pauline Emmett has been involved in the dietary assessment of the Avon Longitudinal Study of Parents And Children (ALSPAC) study since baseline (Emmett, 2009). During the lifetime of this study, dietary assessment of the children's diets has transferred from surrogate parental reports to children themselves reporting their intake. Dr Emmett highlighted the value in using a combination of dietary methods, as the remit of each is different. In ALSPAC the FFQ has been useful in picking up consumption of less commonly eaten foods, whereas food records have been relied on to give individual dietary information. Additional information on the context of eating has been collected. This is an important dimension of dietary assessment, which is particularly valuable in family-based studies.

Dr Emma Foster has been working on a computer-based interactive portion size assessment system (IPSAS) to assist children in portion size estimation (Foster et al., 2009). Children in all age groups using this system have been shown to estimate portion sizes at a level comparable with adults using food photographs. The system is particularly useful to account for leftover food. Further validation work is being undertaken.

Dr Carol Boushey at Purdue University is undertaking pioneering work in the use of technology in dietary 
assessment (Boushey et al., 2009). To date, her work has shown that adolescents prefer methods of dietary assessment that incorporate technology including capturing food images. The rapid advances in technology mean that a diverse team from a range of disciplines is required to develop systems of dietary assessment that may be used on personal digital assistant (PDA) devices and mobile phones. This is an exciting area and is likely to increase the quality of dietary data collected, streamline analysis and improve compliance in this age group, but it presents formidable challenges at this developmental stage.

The remaining three short presentations concentrated on dietary assessment in the elderly. Dr Maria Maynard reported on the long-term follow-up and dietary assessment of the Boyd Orr Cohort (Maynard and Blane, 2009) who were originally surveyed in Britain before the second world war. The FFQ used was a modified version of the EPIC Norfolk FFQ (enlarged font, deleted amalgamated foods, removed portion size information). Missing data were especially problematic in some groups. Qualitative research showed that life transitions, such as death of a spouse, had an important bearing on food habits, with some women reporting a new found freedom in their food choices and men becoming more reliant on convenience foods, illustrating generational and social context issues that impact on food intake.

Dr Elisabet Rothenberg reported on two Swedish studies, which have specifically addressed the validity of dietary reports in elderly by different methods (Rothenberg, 2009). Results indicated overreporting of intake by FFQ compared with food records and urinary nitrogen, whereas diet history showed underestimation when compared with doubly labelled water measurement of total energy expenditure. These results, in addition to data from interviews, indicated that older people in their eighties provided dietary data that was comparable with those obtained in younger adults. Researchers must be mindful of the possibility that reports of diet in older people are related to their diet earlier in life rather than their current diet.

The final short presentation from Dr Jeanne de Vries provided an additional European perspective with experiences of measuring diet in the international HALE, SENECA and FINE studies (de Vries et al., 2009). Researchers in all these studies elected to use the dietary history adapted for use in the elderly and to include a country-specific food checklist. A combination of dietary measures was recommended to assess dietary intake and the challenge of differences in national food composition tables in multicountry studies was highlighted.

The three breakout sessions discussed: portion size estimation, use of surrogate reporters and technological advances in dietary assessment. The challenge of portion size estimation is considerable; the methods used to date were reviewed and it was acknowledged that due to changes in food portion sizes of readily prepared foods (generally an increase), there is an ever increasing disparity with what people are likely to consider an 'average' portion. Thus, utilization of technology such as the computer program being developed at the University of Newcastle or the work being pioneered by Dr Carol Boushey seems to be the way forward. In the meantime, the relative cheapness of digital cameras makes issuing cameras to subjects to photograph their food intake feasible. The use of till receipts may be a useful adjunct to more traditional ways of estimating portion size. Surrogate reporters are necessary in studies of young children or are required to complement a child's report of their own diet, probably up to the age of 10 years. The presence of surrogates may inhibit a child's report and using surrogates who have not been trained in assessment may be problematic. In her presentation, Dr Adamson had cited an example of a study using lay and trained personnel in schools to act as surrogate reporters. Children themselves may act as surrogate reporters, for example, in immigrant families who are unable to speak the language of the country they are resident. Identifying suitable surrogates for older people in a residential setting is much easier than in the community. Again the need for technological advances to improve dietary assessment in those unable to report their food intake was highlighted.

We conclude that the workshop fulfilled its aims and objectives as outlined above. Examples of good practice in dietary assessment in the young and older people were discussed. A thorough examination of the research question and an understanding of the type of dietary data a particular tool will yield are vital at the planning stage of a study. There might be a need to combine the assessment methods; an FFQ, for example, can be useful to elucidate the intake of foods eaten infrequently but regularly, which might be missed by a 4-day food diary, but is not adequate to assess the diet of an individual. Older people represent a diverse population and several presenters showed that older people's ability to report intake might equal that of younger adults but that the presence of cognitive deterioration should be considered and possibly measured. One study has shown the success of multiple-pass 24 -h recalls in this group. Exciting developments using technology in the assessment of diet were presented; some methods are at the development stage and show great promise whereas others are ready to use. Research has advanced knowledge of the cognitive issues in the recall of food intake in children and the target period of recall and order of prompts requires consideration. The influence of ethnicity, parenting and weight status on recall in children requires further research. On a number of occasions the problem in the United Kingdom of not having ready access to a national food composition database, as in the United States and other countries, was cited as a barrier to research.

Dietary assessment is a challenging area of work but developments are being made and technology is likely to advance this field considerably in the future. We would like to take this opportunity to thank all the speakers and audience for participating in the workshop and the speakers 
for willingly contributing papers to this supplement edition of the European Journal of Clinical Nutrition.

\section{Disclosure}

JM Warren was funded by a grant from the MRC's PHSRN. AM Stephen has declared no financial interests.

\section{References}

Adamson AJ, Collerton J, Davies K, Foster E, Jagger C, Stamp E et al. (2009). Nutrition in advanced age: dietary assessment in the Newcastle 85 + study. Eur J Clin Nutr 63 (Suppl 1), S6-S18.

Baxter SD (2009). Cognitive processes in children's dietary recalls: insights from methodological studies. Eur J Clin Nutr 63 (Suppl 1), S19-S32.

Boushey CJ, Kerr DA, Wright J, Lutes KD, Ebert DS, Delp EJ (2009). Use of technology in children's dietary assessment. Eur J Clin Nutr 63 (Suppl 1), S50-S57. de Vries JHM, de Groot LCPGM, van Staveren WA (2009). Dietary assessment in elderly people: experiences gained from studies in the Netherlands. Eur J Clin Nutr 63 (Suppl 1), S69-S74.

Emmett P (2009). Dietary assessment in the Avon Longitudinal Study of Parents and Children. Eur J Clin Nutr 63 (Suppl 1), S38-S44.

Foster E, Adamson AJ, Anderson AS, Barton KL, Wrieden WL (2009). Estimation of portion size in children's dietary assessment: lessons learnt 63 (Suppl 1), S45-S49.

Maynard MJ, Blane D (2009). Dietary assessment in early old age: experience from the Boyd Orr Cohort. Eur J Clin Nutr 63 (Suppl 1), S58-S63.

McNeill G, Winter J, Jia X (2009). Diet and cognitive function in later life: a challenge for nutrition epidemiology. Eur J Clin Nutr 63 (Suppl 1), S33-S37.

Rothenberg EM (2009). Experience of dietary assessment and validation from three Swedish Studies in the elderly. Eur J Clin Nutr 63 (Suppl 1), S64-S68.

JM Warren and AM Stephen Department of Population Nutrition Research, MRC Human Nutrition Research, Elsie Widdowson Laboratory, Cambridge, UK E-mail: alison.lennox@mrc-hnr.cam.ac.uk 